\title{
Analysis of the Influence of Meteorological Factors on GPS Tropospheric Delay Correction
}

\author{
YIN Longlong \\ University of Chinese Academy of Sciences, Beijing \\ National Time Service Centre, Chinese Academy of Sciences, Xi'an \\ Key Laboratory of Time and Frequency Standards, Chinese Academy of Sciences \\ YUAN Haibo, DONG Shaowu \&ZHANG Jihai \\ National Time Service Centre, Chinese Academy of Sciences, Xi'an \\ Key Laboratory of Time and Frequency Standards, Chinese Academy of Sciences
}

\begin{abstract}
Tropospheric delay is one of the important errors in positioning and timing. Lots of researches have been done, and many tropospheric correction models have been proposed. The precision of the models change with the location and meteorological conditions of the user. Significant improvements will been made within the precision of positioning and timing on researching tropospheric correction models. In this paper, the effects of different factors on the precision of tropospheric correction models are analyzed, and their proportions in the measurement uncertainty are elaborated. Based on the two years ZTD (zenith tropospheric delay) of BJFS station, the effects of meteorological parameters on the zenith delay correction are analyzed, and the precision of various models are compared. The result shows that the precision of models are the highest by using direct meteorological measurements, and the precision of ENGONS and UNB3 follow, and the models using reference atmospheric information get poor precision.
\end{abstract}

KEYWORD: Tropospheric delay; Meteorological parameters; GPS timing

\section{INTRODUCTION}

The troposphere is non-dispersive medium, and the influence on the GNSS signal is non-dispersive refraction. Its transmission path is not a straight line, and the velocity changes. The refractive index of the electromagnetic wave, which has no relation with the frequency or wavelength, is just associated with the propagation velocity [1].

The effect of troposphere on time transfer delay reaches $9 \mathrm{~ns}$ in the zenith direction. Generally, zenith delay error is $0.2 \sim 3 \mathrm{~ns}$ when corrected by tropospheric correction models. $90 \%$ of tropospheric delay is caused by the dry term of atmosphere, while the remaining $10 \%$ is aroused by the wet term. The dry term is mainly concerned with atmospheric temperature and pressure, while the wet term is associated with water vapor pressure. At present, the accuracy of correction model for dry term delay attains $90 \%$ or more, while the accuracy for wet term delay can only reach $20 \%$ [2]. Tropospheric delay models have been applied to lots of engineering practices. However, when positioning and timing accuracy is high, the tropospheric delay is still the main error source.

In the paper, firstly, the calculation of meteorological parameters is introduced. Secondly, the proportions of the factors which are accounted for in the measurement uncertainty are elaborated by calculating the factors (pressure, temperature and water vapor pressure, elevation, etc.) which affect the accuracy of models. Lastly, the correction precision of various models are compared and the result is given.

\section{MODELS INTRODUCE}

\subsection{Hopfield model}

The model was proposed by Hopfield in 1969 by averaging one year data of 18 stations around the world. The decreasing rate of atmospheric temperature is assumed to be a constant $\left(\beta=6.8^{\circ} \mathrm{C} / \mathrm{km}\right)$. See for Equation 1 below: [3][5]

$$
\left\{\begin{array}{l}
\delta \rho_{\text {trop }}=\delta \rho_{d}+\delta \rho_{w} \\
\delta \rho_{d}=\frac{\delta S_{d}}{\sin \left(E^{2}+6.25\right)^{1 / 2}} \\
\delta \rho_{w}=\frac{\delta S_{w}}{\sin \left(E^{2}+2.25\right)^{1 / 2}} \\
\delta S_{d}=155.2 \times 10^{-7} \frac{P_{s}}{T}\left(h_{d}-h_{s}\right) \\
\delta S_{w}=7.46512 \times 10^{-2} \frac{e_{s}}{T_{s}^{2}}\left(h_{w}-h_{s}\right) \\
h_{d}=40136+148.72\left(T_{s}-273.16\right) \\
h_{w}=11000
\end{array}\right.
$$


Where $\delta \rho_{\text {trop }}=$ tropospheric delay on the transmission path; $\delta \rho_{d}=$ dry term on the transmission path; $\delta \rho_{w}=$ wet term on the transmission path; $\delta S_{d}=$ dry term delay on the zenith; $\delta S_{w}=$ wet term delay on the zenith; $E=$ satellite elevation angle (in degrees), $h_{d}=$ top height of dry atmospheric air; $h_{w}=$ top height of wet atmospheric air; $h_{s}=$ station elevation (in meter), $P_{s}$ $=$ station pressure (in mbar); $T_{s}=$ station temperature (in degrees Kelvin); and $e_{s}=$ water vapor pressure (in mbar).

\subsection{Saastamoinen model}

In 1983, Bauersima put forward Saastamoinen model. See for Equation 2 below: [4][5]

$\left\{\begin{array}{l}\delta=\frac{0.002277}{\sin E}\left[P_{s}+\left(\frac{1255}{T_{s}}+0.05\right) e_{s}-B \cot E\right] W(\varphi, H)+\delta R \\ W(\varphi, H)=1+0.0026 \cos 2 \varphi+0.0028 h_{s}\end{array}\right.$

Where $\delta=$ tropospheric delay on the transmission path; $E=$ satellite elevation angle (in degrees); $h_{s}=$ station elevation (in meter); $P_{s}=$ station pressure (in mbar); $T_{s}=$ station temperature (in degrees Kelvin); $e_{s}=$ water vapor pressure (in mbar); $B$ is a list function of $h_{s}$, and $\delta R$ is a list function of $h_{s}$ and $E$ [12].

The simplification of the above formula is:

$$
\left\{\begin{array}{l}
\delta=\frac{0.002277}{\sin E^{\prime}}\left[P_{s}+\left(\frac{1255}{T_{s}}+0.05\right) e_{s}-\frac{a}{\tan ^{2} E}\right] \\
E^{\prime}=E+\Delta E \\
\Delta E=\frac{16^{\prime \prime}}{T_{s}}\left(P_{s}+\frac{4810}{T_{s}} e_{s}\right) \cot E \\
a=1.16-0.15 \times 10^{-3} h_{s}+0.716 \times 10^{-8} h_{s}
\end{array}\right.
$$

Where the meanings of the parameters are the same as the previous.

\subsection{Other correction models}

Simple tropospheric correction model [5], which only needs inputs of station elevation and satellite elevation, has the accuracy of $92 \% \sim 95 \%$.

Rodrigo Leandro et al from Canada New Brunswick University established UNB (University of New Brunswick) model [6][7][8]. The model accounts for the variety of meteorological parameters with the season which is reflected by the day of year. The model first obtains ZTD at the sea level, then adds station latitude, elevation and other location conditions, and gets the ZTD at the station at last.
The European Geo-stationary Navigation Overly System adopts the EGNOS model which is not affected by meteorological parameters. The day of year, station latitude and elevation are the impact factors of the ZTD of the model. Among them, day of year can reflect temperature, pressure, relative humidity and other parameters in UNB model, but also covers the influence of temperature gradient and moisture gradient. At standard atmosphere, EGNOS gets higher precision than UNB model [8][9][10].

\subsection{Meteorological parameters}

In the condition of indirect meteorological measurements, the pressure, temperature and relative humidity can be derived by the meteorological model which are associated with elevation of station [12].

$$
\left\{\begin{array}{l}
P_{s}=P_{0}\left(1-0.000226 h_{s}\right)^{5.225} \\
T_{s}=T_{0}-0.0065 h_{s} \\
R_{h}=R_{h 0} \exp \left(-0.0006396 h_{s}\right) \\
e_{s}=R_{h} \times \exp \left(-37.2465+0.213166 T_{s}-0.000256908 T_{s}^{2}\right)
\end{array}\right.
$$

Where $h_{s}=$ station elevation (in meter); $P_{0}=$ $1013.25 ; T_{0}=288.15 \mathrm{~K} ; R_{h 0}=0.5 ; P_{s}=$ station pressure (in mbar); $T_{s}=$ station temperature (in degrees Kelvin); and $e_{s}=$ water vapor pressure (in mbar);

In the condition of direct meteorological measurements, water vapor pressure $e_{s}$ can be obtained by $\mathrm{RH}$ (relative humidity) or station dry temperature and wet temperature while $P_{s}$ and $T_{s}$ can be measured directly. See for Equation 5 below: [13]

$$
\left\{\begin{array}{l}
e_{s}=e_{w}-4.5 \times 10^{-4} \times\left(1+1.68 \times 10^{-3} T_{w}\right) \times\left(T_{s}-T_{w}\right) \times P_{s} \\
e_{w}=1013.246 \times\left(\frac{373.16}{T_{w}}\right)^{5.02808} \times \exp \left(-g\left(T_{w}\right)\right) \\
g\left(T_{w}\right)=g_{1}\left(T_{w}\right)+g_{2}\left(T_{w}\right)+g_{3}\left(T_{w}\right) \\
g_{1}\left(T_{w}\right)=18.19728 \times\left(\frac{373.16}{T_{w}}-1\right) \\
g_{2}\left(T_{w}\right)=0.0187265 \times\left\{1-\exp \left[-8.03945 \times\left(\frac{373.16}{T_{w}}-1\right)\right]\right\} \\
g_{3}\left(T_{w}\right)=3.1813 \times 10^{-7} \times \exp \left[-26.1205 \times\left(\frac{373.16}{T_{w}}-1\right)-1\right]
\end{array}\right.
$$

\section{ANALYSIS OF MODEL ERROR}

When the measurement result is affected by many factors to form a plurality of uncertainty components, the uncertainty of measurement result 
can be obtained by combining each standard uncertainty component [11]. Such as in indirect measurement, the estimated value y of measured $\mathrm{Y}$ is obtained by other measurements $x_{1,} x_{2, \cdots} x_{N}$. That is:

$$
y=f\left(x_{1}, x_{2}, \cdots x_{N}\right)
$$

Formula for the measurement uncertainty is:

$$
\sigma=\sqrt{\sum_{i=1}^{N}\left(\frac{\partial f}{\partial x_{i}}\right)^{2}\left(u_{x i}\right)^{2}+2 \sum_{1 \leq i \leq j}^{N} \frac{\partial f}{\partial x_{i}} \frac{\partial f}{\partial x_{j}} \rho_{i j} u_{x i} u_{x j}}
$$

Where $u_{x i}=$ uncertainty of $x_{i}$ (direct measurement), $\rho_{i j}=$ the correlation of any two directly measured values; If $x_{i}$ and $x_{j}$ are independence, $\rho_{i j}=0$.

To analyze the influence of meteorological parameters on tropospheric delay, this paper selects two years meteorological observation data of BJFS (2012 2013). Figure 1-4 are tendencies of pressure, temperature, relative humidity, water vapor pressure, respectively. The result bellow is obtained by calculating the data: the correlation between pressure and temperature is -0.823024 ; the correlation between pressure and water vapor pressure is 0.740459; the correlation between the temperature and water vapor pressure is 0.77950 ; the correlation between pressure and relative humidity is -0.3084 ; the correlation between temperature and relative humidity is 0.6218; and the correlation between water vapor pressure and relative humidity is 0.623658 .

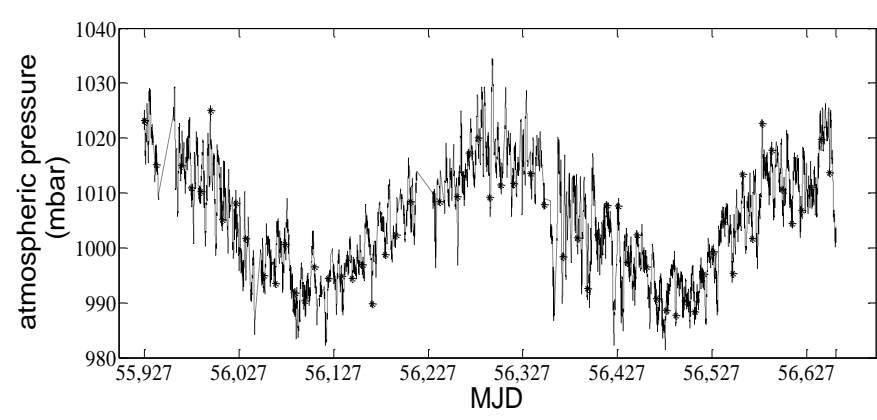

Figure 1. 2012.1-2013.12 pressure variation

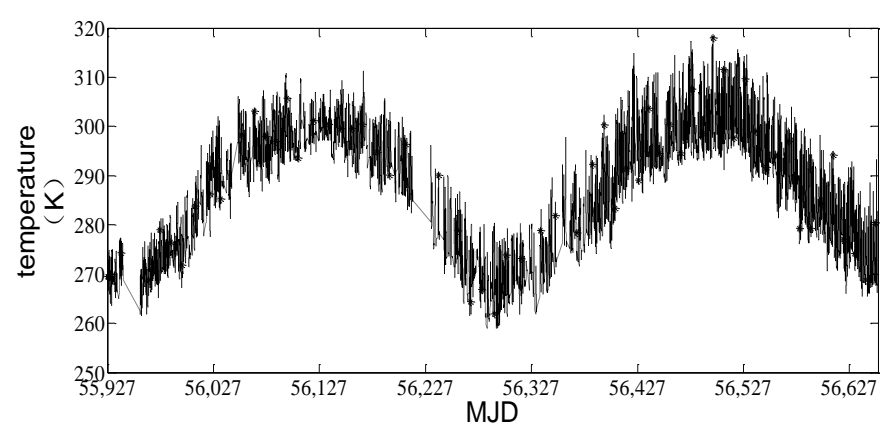

Figure 2. 2012.1-2013.12 temperature variation

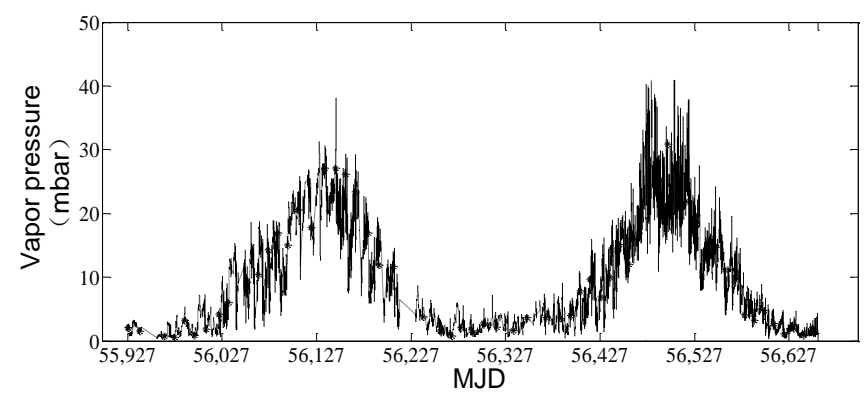

Figure 3. 2012.1-2013.12 water vapor pressure variation

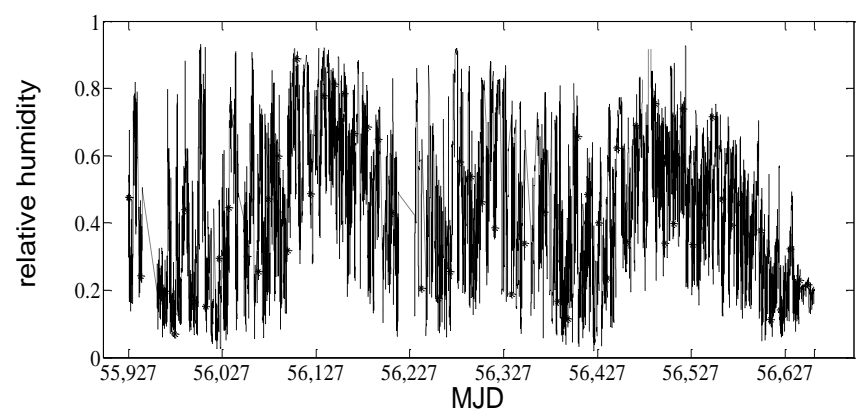

Figure 4. 2012.1-2013.12 relative humidity variation

In the calculation, the parameters are assumed at standard atmospheric pressure. The correlation between two factors have been given above. Others are zero. $P_{0}=1013.25 ; T_{0}=288.15 \mathrm{~K} ; R_{h 0}=0.5$; $h=87.46 \mathrm{~m} ; \varphi=39.6^{\circ}$. For the result, see for Equation 8-10 below:

$\sigma_{d}^{h o p}=\sqrt{0.002277^{2} u_{P}^{2}+0.000109^{2} u_{T}^{2}+0.000055^{2} u_{h}^{2}-0.00000224 u_{P} u_{T}}$

$\sigma_{w}^{\text {hop }}=\sqrt{0.009811^{2} u_{e}^{2}+0.000586^{2} u_{T}^{2}+0.000008^{2} u_{h}^{2}-0.000033 u_{P} u_{e}}$

$\left\{\begin{array}{l}\sigma_{z}^{\text {hop }}=\frac{1}{100} \sqrt{0.2277^{2} u_{P}^{2}+0.0596^{2} u_{T}^{2}+0.9811^{2} u_{e}^{2}+0.0056^{2} u_{h}^{2}+\varepsilon} \\ \varepsilon=-0.02235 u_{P} u_{T}-0.3308 u_{P} u_{e}+0.0912 u_{T} u_{e}\end{array}\right.$

So, in Hopfield model, for the dry term delay, pressure measurement error accounts for the main, followed by the temperature measurement error, elevation measurement error with minimal impact. For the wet term delay, water vapor pressure measurement error accounts for the main, the temperature measurement error secondly, and the effect of elevation measurement error is minimum.

\section{EXPERIMENT AND RESULTS}

Influence of satellite elevation angle on the link delay is analyzed by selecting the G18 satellite (elevation angle varies from 5 to 89 degrees at BJFS station). As shown in Figure 5, the delay introduced 
by link reaches 70ns when the satellite is at low elevation angle. With the elevation angle increasing, the delay decreases gradually. By using BJFS direct meteorological measurements and ZTD on January 2, 2013 published by IGS, the difference between the delay on the path to G18 modified by different models and BJFS ZTD (published by IGS) mapping to the path to G18 is calculated. In Figure 6, at low elevation angle, each model introduces a relatively large error about $4 \mathrm{~ns}$. So smaller elevation leads to larger error on its path.

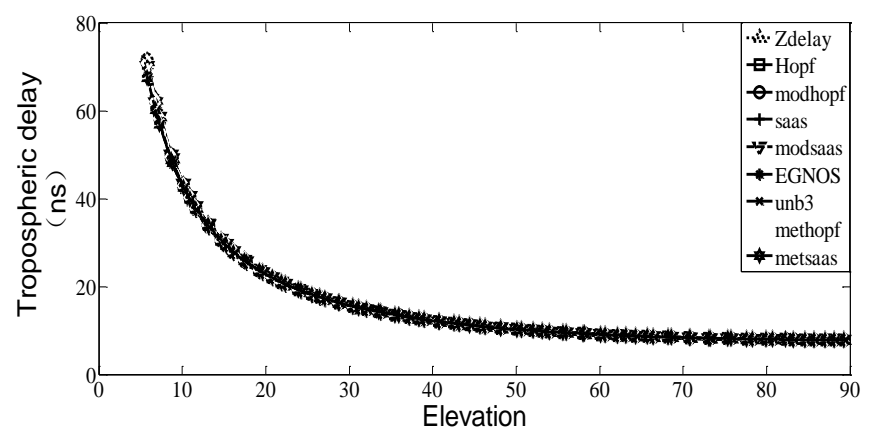

Figure 5. Influence of the satellite elevation angle on tropospheric delay

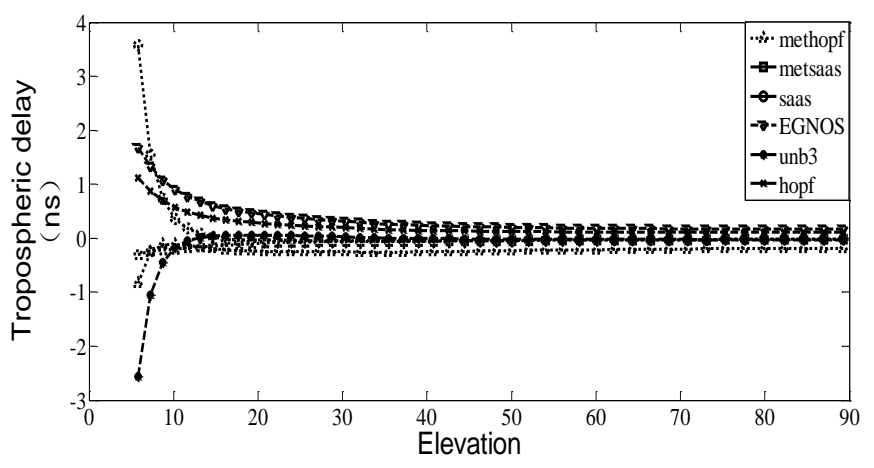

Figure 6. Influence of the satellite elevation angle on tropospheric delay

A year of volatility of BJFS ZTD (published by IGS) is greater than 1ns. To compare and analyze the effect of meteorological factors on the troposphere, this paper chooses BJFS two years meteorological and ZTD (published by IGS). See for Figure 7, 8.

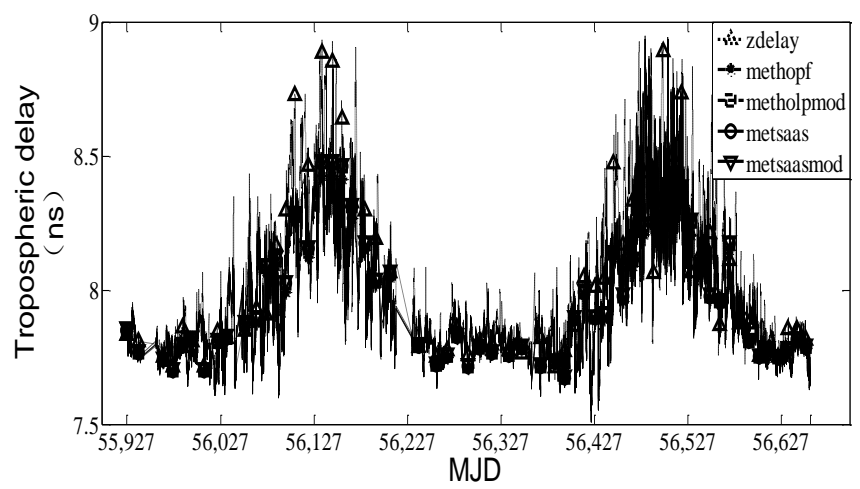

Figure 7 Comparison between models with direct meteorological measurements in zenith (BJFS 2012-2013)

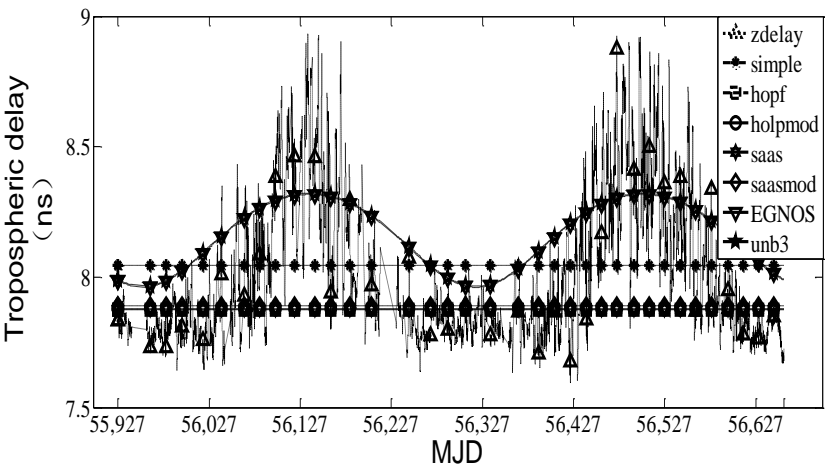

Figure 8 Comparison between models with reference atmospheric information in zenith (BJFS 2012-2013)

In Figure 7, where Zdealy is ZTD (published by IGS), methopf is Hopfield (direct meteorological measurements), methopfmod is modification Hopfield (direct meteorological measurements), metsaas is Saastamoinen (direct meteorological measurements), metsaasmod is modification Saastamoinen (direct meteorological measurements). The range of ZTD a year of fluctuations is 7.7ns 9ns. Tropospheric delay is associated with temperature, pressure, relative humidity and water vapor pressure, with the correlation being given before. The consistency of ZTD of models (direct meteorological measurements) and ZTD published by IGS is better, and each model correction effect has little difference.

In Figure 8, where Zdealy is ZTD published by IGS, simple is simple troposphere correction model, Hopf and hopfmod are Hopfield model and modification-Hopfield model respectively, SaaS and saasmod are Saastamoinen model and modification Saastamoinen model respectively. According to the characteristics of models, under the condition of fixed station coordinate and using reference atmospheric information, the correction results are constants while the results of EGNOS and UNB3 two global tropospheric models correction are sine functions of time. As seen from Figure 8, the correction effects of these two models which reflect the ZTD (published by IGS) are basically the same. See for Table 1, 2.

Table 1. In 2012, the RMS and standard deviation of models.

\begin{tabular}{|l|c|c|}
\hline \multirow{2}{*}{\multicolumn{1}{|c|}{ model }} & RMS & STDEV \\
\cline { 2 - 3 } & $\mathrm{ns}$ & $\mathrm{ns}$ \\
\hline simple & 0.277037 & 0.274955 \\
\hline Hopfield & 0.307701 & 0.274955 \\
\hline Mod Hopfield & 0.305476 & 0.274955 \\
\hline Saastamoinen & 0.305476 & 0.274955 \\
\hline Mod Saastamoinen & 0.301250 & 0.274955 \\
\hline EGNOS & 0.248039 & 0.199697 \\
\hline UNB3 & 0.250459 & 0.199672 \\
\hline Hopfield(Metrologic) & 0.137143 & 0.117722 \\
\hline Mod Hopfield (Metrologic) & 0.125814 & 0.112653 \\
\hline Saastamoinen(Metrologic) & 0.125814 & 0.112653 \\
\hline Mod Saastamoinen(Metrologic) & 0.121688 & 0.112653 \\
\hline
\end{tabular}


Table 2. In 2013, the RMS and standard deviation of models.

\begin{tabular}{|l|c|c|}
\hline \multirow{2}{*}{ model } & RMS & STDEV \\
\cline { 2 - 3 } & $\mathrm{ns}$ & $\mathrm{ns}$ \\
\hline simple & 0.281741 & 0.281183 \\
\hline Hopfield & 0.320745 & 0.281183 \\
\hline Mod Hopfield & 0.318356 & 0.281183 \\
\hline Saastamoinen & 0.318356 & 0.281183 \\
\hline Mod Saastamoinen & 0.313788 & 0.281183 \\
\hline EGNOS & 0.249131 & 0.206014 \\
\hline UNB3 & 0.251426 & 0.205986 \\
\hline Hopfield(Metrologic) & 0.173066 & 0.143785 \\
\hline Mod Hopfield (Metrologic) & 0.163280 & 0.141335 \\
\hline Saastamoinen(Metrologic) & 0.163280 & 0.141335 \\
\hline Mod Saastamoinen(Metrologic) & 0.158506 & 0.141335 \\
\hline
\end{tabular}

The RMS and STDEV of models with direct meteorological measurements are relatively small, followed by that of EGNOS and UNB3 models whose accuracy of zenith correction delay are $0.2 \mathrm{~ns}$. The precision of simplified model is poorer than that of the EGNOS and UNB3, but higher than that of the model with reference atmospheric information.

\section{CONCLUSIONS}

The impact of meteorological factors on tropospheric delay correction is evident. ZTD calculated by model with direct meteorological measurements and ZTD published by IGS are closest. The precision of ENGONS and UNB3 models follow, and the models using reference atmospheric information get poor precision. Since UNB3 and EGNOS do not need the measured meteorological parameters, with a certain precision, they could be used as correction model of real time transfer.

In short baseline time transfer, since the meteorological conditions of the signal transmission path from satellite to the both ends of baseline are similar, most error of tropospheric delay could be eliminated by using the correlation of delay. In long baseline time transfer, changing the cut-off angle and adding the measured parameters can improve time transfer accuracy. In actual calculation, the user can choose a suitable model to correct the tropospheric delay according to observation environment and conditions.

\section{REFERENCES}

[1] Chen Zhaohua. 2010. Regional precise tropospheric delay modeling. Hunan: Central South University,

[2] Zhang Shuangcheng \& Zhang Pengfei \& Fan Pengfei. 2012. Recent progress and comparative analysis of tropospheric correction models based on GPS Geodesy and Geodynamics, 32 (2): 92-95.

[3] Hopfield H S. 1971. Tropospheric effect on electrometrically measured range: prediction from surface weather data. Rad Sci. (6): 357 - 367

[4] Saastamoinen J. 1972. Introduction to practical computation of astronomical refraction. Journal of Geodesy. 106 (1): 383-397.

[5] Ren Yafei. 2006. An Analysis of Troposphere Delay in GPS Positioning Error. Shaanxi: Journal of Xi'an University. 22 (4): 408

[6] Leandro R F \& Santos M C \& Langley R B. 2006. UNB neutral atmosphere models: Development and performance (A). Proceedings of ION NTM Monterey, California, 564-573.

[7] Zhou Mingduan \& Guo Jiming \& Meng Xiangguang. 2008. GPS tropospheric delay model UNB3 and its accuracy analysis. Wuhan: Journal of Geomatics. 33 (4): 3-5.

[8] Qu Wei Jing \& Zhu Wenyao \& Song Shuli. 2008. The Evaluation of Precision about Hopfield, Saastamoinen and EGNOS Tropospheric Delay Correction Model. acta Astronomica Sinica.49 (1): 113 - 122

[9] Penna N \& Dodson A \& Chen W. 2001. Assessment of EGNOS tropospheric correction model. The Journal of Navigation. 54: 3755.

[10] Liu Jingye \& Song Yuanming \& Hu Jiaxing. 2011. EGNOS a Tropospheric delay correction model and its accuracy analysis. Geospatial information.9 (2): 96-98.

[11] Fei Yetai. 2010. Error theory and data processing (Sixth Edition). Beijing: Mechanical Industry Press.

[12] Xu Guochang 2011. GPS theory, algorithms and Applications (Second Edition). Beijing. Tsinghua University press,

[13] Wang Xinlong \& Li Yafeng. 2008. Applicability analysis of four sorts of troposphere delay error models in GPS positioning. Beijing: Electronics Optics \& Control. 15 (11): 6-9. 